\title{
Pointes liquides
}

Élise Lorenceau ${ }^{1}$, Étienne Reyssat ${ }^{2}$, Frédéric Restagno ${ }^{3}$,

David Quéré2 (david.quere@espci.fr)

1 - Laboratoire de Physique des Milieux Divisés et des Interfaces, Université de Marne-la-Vallée

2 - Physique et Mécanique des Milieux Hétérogènes, ESPCI, Paris

3 - Laboratoire de Physique des Solides, Université Paris XI, Orsay

Nous décrivons ici ce

qui se passe lorsqu'un

liquide visqueux est

amené à se rencontrer

lui-même, comme

quand on verse de

l'huile dans un verre qui

en contient déjà,

ou quand on bat

des œufs en neige.

Si l'impact n'est pas

trop violent, on fabrique

à l'endroit

de la rencontre

des liquides une zone

remarquable,

parfaitement pointue

à l'œil nu.

Si le choc est plus fort,

ces pointes montrent

leur fragilité en cédant :

de l'air est entraîné avec

le liquide en mouvement,

conduisant à la

formation d'une mousse.
L'existence, à la surface des liquides, d'une tension dite superficielle a pour conséquence de régulariser ces surfaces. Puisque l'interface entre le liquide et le milieu extérieur porte une énergie, et parce que les liquides sont déformables, une goutte, une bulle, un bain prendront la forme qui minimise leur surface, compte tenu des contraintes supplémentaires qui peuvent s'exercer sur le liquide (souvent, la gravité). Dans ces conditions, les surfaces liquides sont lisses (jusqu'à l'échelle le plus souvent nanométrique où les fluctuations thermiques sont susceptibles de les perturber), ce que l'industrie du verre, par exemple, a su admirablement exploiter : en coulant du verre en fusion sur de l'étain fondu dans une atmosphère d'azote, on fabrique des plaques de verre lisses jusqu'à l'échelle de quelques angströms.

Il existe cependant des situations où, sous l'effet d'une contrainte localisée, une surface liquide se déforme de façon extraordinaire, jusqu'à produire une forme improbable par excellence sur une telle surface, à savoir une pointe [1]. On pourrait imaginer qu'il faut pour cela des circonstances extrêmes, mais non : un acte aussi simple que de verser de l'huile d'olive dans un bol qui contient déjà un fond de cette huile engendre, à l'endroit où le jet rencontre le bain, un creux dont la base forme une pointe acérée. Pour étudier le phénomène, la géométrie du jet est cependant malcommode, car elle nous empêche, quand on regarde de profil, de distinguer ce qui se passe exactement à l'endroit de l'impact.

\section{Où l'on creuse un liquide}

Aussi a-t-on imaginé de travailler avec un rouleau solide partiellement immergé dans un bain d'huile, contenu lui-même dans un réservoir transparent. Quand le rouleau tourne, il entraîne d'un côté de l'huile qui de l'autre vient frapper le bain à une vitesse bien contrôlée [2]. Si on observe de profil ou de dessous la zone d'impact, on distingue mieux ce qui s'y passe (fig. 1). À faible vitesse d'impact d'abord (typiquement quelques centimètres par seconde pour des huiles 100 à 1000 fois plus visqueuses que l'eau), le choc creuse une sorte de trou stationnaire dont la profondeur augmente avec la vitesse, et qui peut s'enfoncer d'un bon centimètre dans le bain. Mais le point le plus remarquable concerne la base de ce trou : on y distingue une pointe (une pointe d'air dans de l'huile), qui est d'autant plus marquée que la vitesse d'impact est grande, et qui à l'œil nu ressemble à un point de rebroussement. À plus grande vitesse, la base de la pointe cède et un film d'air (qui réfléchit la lumière dans la figure $1 \mathrm{~b}$ ) s'engouffre avec le liquide en mouvement. Nous décrivons ici ces deux situations bien différentes.
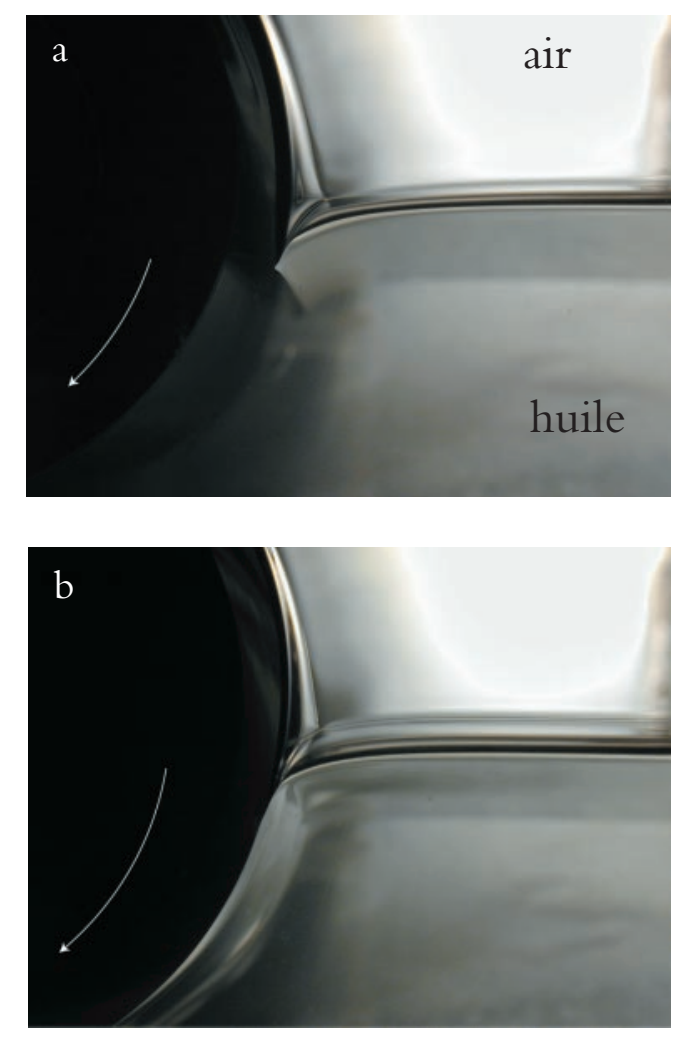

Figure 1 : Rouleau solide immergé dans une huile silicone mille fois plus visqueuse que l'eau. L'ensemble est contenu dans un récipient transparent, ce qui permet de prendre des vues de profil. Le rouleau, qui tourne dans le sens des aiguilles d'une montre à $10 \mathrm{~cm} / \mathrm{s}$ (comme l'indique la flèche), entraîne de l'huile d'un côté qui de l'autre frappe le bain. À cet endroit, il se forme un creux dont la base est une pointe acérée (a). Si le rouleau tourne plus vite $(20 \mathrm{~cm} / \mathrm{s})$, la pointe craque et un film d'air s'engouffre dans le bain (b).

À l'aide de macrophotographies, on peut mesurer le rayon de courbure en bout de pointe [2]. On voit sur la figure 2 (où l'air apparaît en noir et l'huile en gris clair) qu'il est une fonction très sensible de la vitesse, puisque sa valeur passe 

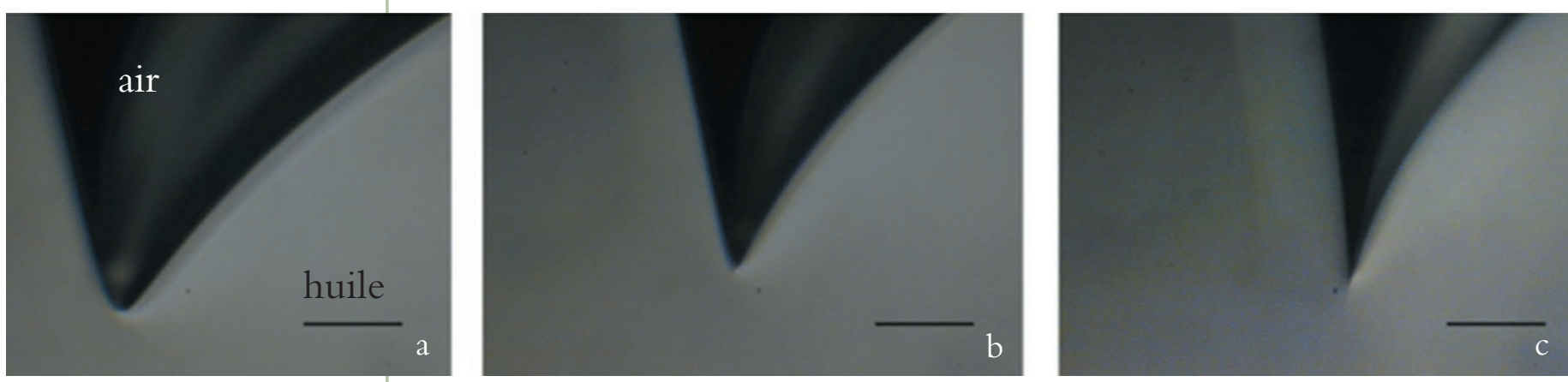

Figure 2. Macrophotographies des pointes liquides stationnaires engendrées par la rencontre d'un liquide 1000 fois plus visqueux que l'eau avec lui-même, à $10 \mathrm{~cm} / \mathrm{s}(a)$, $12 \mathrm{~cm} / \mathrm{s}$ (b) et $15 \mathrm{~cm} / \mathrm{s}$ (c). La barre indique 200 microns et les pointes sont photographiées à travers la vitre du récipient contenant l'huile, dans l'expérience des rouleaux décrite sur la figure 1. de 100 à 1 micron quand la vitesse augmente de 10 à $15 \mathrm{~cm} / \mathrm{s}$. Pour être plus précis, on trouve que ce rayon décroît exponentiellement avec la vitesse. Ceci signifie qu'il existe une vitesse caractéristique au-delà de laquelle la courbure de la pointe diverge. On peut être tenté de construire cette vitesse à partir de deux caractéristiques du liquide, qui sont sa tension de surface $\gamma$ et sa viscosité $\eta$ (voir l'encadré). Or le simple rapport $\gamma / \eta$ de ces deux grandeurs a justement la dimension d'une vitesse, caractéristique du liquide, et qui est celle qui fixe la dynamique de tout processus mû par la tension superficielle et modéré par la viscosité.

On est ainsi tenté de mesurer les rayons de courbure de ces pseudo-pointes en fonction du nombre capillaire $C a=\eta V / \gamma$, qui compare la vitesse $V$ de l'impact à la vitesse $\gamma / \eta$ caractéristique du liquide. La figure 3 montre que la pointe devient acérée lorsque le nombre capillaire est d'ordre unité mais, mieux encore, que l'ensemble des résultats (pour des systèmes variés où l'on est allé jusqu'à remplacer l'air par une huile légère) suit une même courbe, qui est une exponentielle.

Dans un article classique de 1992, Jeong et Moffatt ont prédit une telle dépendance, et un argument de John Hinch permet de comprendre très simplement leur résultat [3]. La pointe est comme un cylindre (ou un demicylindre) qui remonte à la vitesse - $V$ le liquide descendant à la vitesse $V$ (ainsi elle apparaît fixe, à nous qui la regardons). C'est la tension superficielle $\gamma$ qui tire sur la pointe (pour la résorber) et lui donne son mouvement. Or, la force de friction visqueuse sur un cylindre de rayon $r$, qui est l'équivalent à deux dimensions du problème classique de Stokes d'une sphère tirée dans un bain, s'écrit - $\eta V / \ln r$ (où $r$ doit être normé par une longueur externe, qui

\section{Pointes, tension de surface et viscosité}

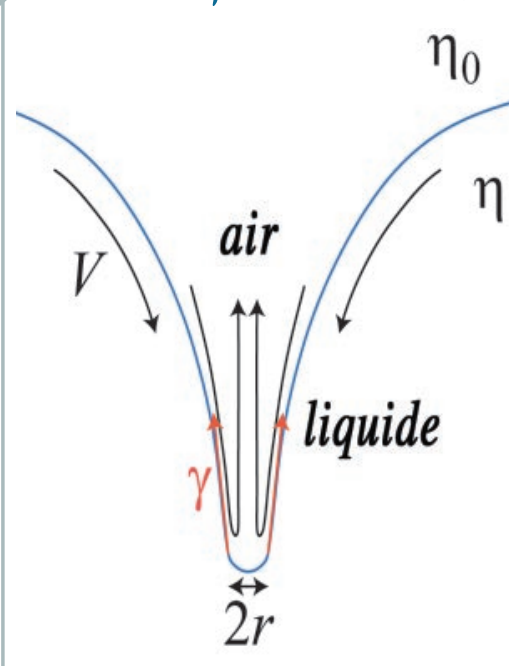

Pointe d'air dans un liquide de viscosité $\eta$ amené à se heurter luimême à une vitesse $V$. On note $r$ le rayon de la pointe (qui méritera ce nom si r est inférieur à 100 microns), et $\gamma$ la force de tension superficielle qui tend à faire remonter la pointe. $\eta_{0}$ est la viscosité du fluide supérieur (de l'air, ici).
Un liquide est caractérisé (entre autres) par sa tension de surface $\gamma$, sa viscosité dynamique $\eta$ et sa densité $\rho$. La tension de surface, reflet de la cohésion moléculaire du liquide, traduit l'élasticité de sa surface : si l'on distord une interface, le liquide (qui s'oppose à l'augmentation de la surface) résiste avec une énergie $\gamma$, par unité de surface déformée. $\gamma$ s'exprime donc en $\mathrm{N} / \mathrm{m}$, comme la raideur d'un ressort.

La viscosité d'un liquide (ou d'un gaz) traduit sa résistance à l'écoulement. La formule la plus célèbre de I'hydrodynamique a trait à la viscosité : c'est la force de traînée sur une sphère solide (de rayon $R$ ) que l'on tire (à vitesse $V$ ) dans un liquide de viscosité $\eta$. Cette force, calculée par Stokes, vaut $6 \pi \eta R V$. Ainsi la quantité $\eta V$ apparaît-elle également comme une force par unité de longueur, si bien que le rapport $\eta V / \gamma$ n'a pas de dimension : en d'autres termes, la quantité $\gamma / \eta$ est une vitesse, caractéristique d'un fluide donné - la vitesse naturelle d'un mouvement lié à la tension de surface et ralenti par la viscosité.

Il existe aussi une formule analogue pour un cylindre que l'on déplace dans un liquide. Alors, la force de traînée, écrite par unité de longueur du cylindre, vaut $\eta V /$ In $r$ : cette force s'annule avec le rayon du cylindre, mais d'une façon " pathologique " (en 1/lnr). Il conviendrait, bien entendu, de diviser dans cette formule le rayon $r$ par une taille caractéristique, mais cette taille peut dépendre du problème (ce peut être la longueur du cylindre, ou une longueur hydrodynamique, ou une autre distance caractéristique de la géométrie considérée).

Nous décrivons ici comment des pointes liquides peuvent apparaître à une interface sous l'effet d'un impact, et décrivons leur stabilité. Nous avons schématisé dans la figure ci-contre la géométrie liée à cette situation. On y voit le détail de la pointe visible sur la figure 1a. Le rayon de courbure au bas de la pointe est noté $r$; cette région est tirée vers le haut par la tension de surface, de façon à réduire l'aire de l'interface liquide/vapeur. On peut se représenter le mouvement de cette ligne (la pointe est invariante dans la direction perpendiculaire à la figure) comme celui d'un (demi) cylindre avançant à vitesse $V$ dans un liquide visqueux.

L'écoulement à l'intérieur de la pointe doit également être pris en compte : l'air (ou toute autre phase surnageante) est entraîné par le mouvement du liquide visqueux dans la pointe, dont il ressort comme indiqué par les flèches. On peut définir une pression (dite de lubrification) associée à la contrainte visqueuse engendrée par cet écoulement, qui est proportionnelle à la viscosité $\eta_{0}$ de cette phase (de l'air, le plus souvent). Même si la viscosité de l'air est faible, le fort confinement dans la région de la pointe peut être responsable de la rupture de cette pointe : alors de l'air s'engouffre dans le bain, comme on le voit sur les figures 1 b et $4 b$. 
dépend de la géométrie de l'expérience). L'équilibre entre ces deux forces, qui sont toutes deux exprimées par unité de longueur du cylindre, s'écrit : $\gamma \sim-\eta V / \ln r$. Cette équation implique bien une décroissance exponentielle pour la courbure de la pointe $(r \sim \exp (-\eta V / \gamma))$, et ce, en fonction du nombre capillaire $C a=\eta V / \gamma$.

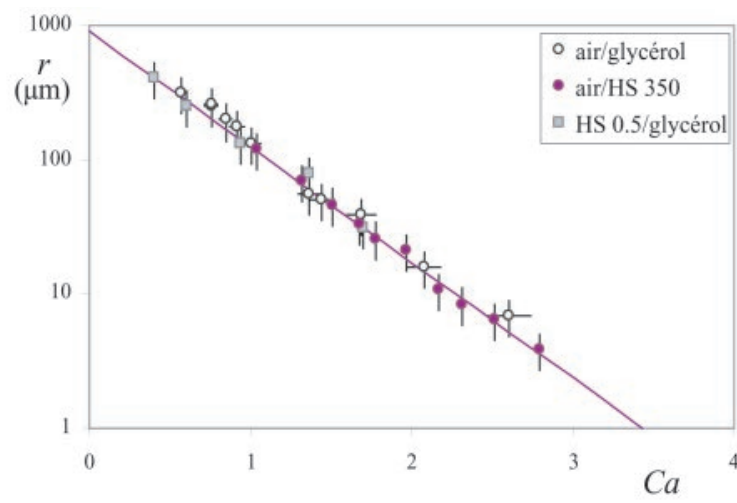

Figure 3 : Rayon d'une pointe d'air (ou d'une huile légère) dans du glycérol (ou une huile visqueuse), produite à la base de la figure d'impact d'une lame de glycérol sur lui-même. Le rayon de la pointe est porté en coordonnées semilogarithmiques en fonction du nombre capillaire $\mathrm{Ca}=\eta \mathrm{V} / \gamma$ défini à partir des viscosité $\eta$ et tension de surface $\gamma d u$ glycérol (ou de l'huile visqueuse) et de la vitesse $\mathrm{V}$ de l'impact. Les mentions HS sont pour huile silicone, avec des viscosités égales à 0,5 ou à 350 fois celle de l'eau.

\section{Où la pointe craque}

Une telle loi ne peut survivre à une augmentation de la vitesse d'impact. Quand le nombre capillaire, argument de l'exponentielle, devient supérieur à l'unité, le rayon de courbure décroît vertigineusement, jusqu'à atteindre (théoriquement) des valeurs subatomiques. On devine qu'il n'en est rien. Quand le nombre capillaire atteint une valeur de quelques unités, l'interface craque, littéralement, et de l'air est entrainé (figure 1b). L'interprétation physique de ce phénomène est encore l'objet de controverses, mais un scénario très plausible a été proposé il y a cinq ans par Jens Eggers, de l'Université de Bristol [4]. L'idée est la suivante : sous le seuil d'entraînement, l'air qui entre dans la pointe (tiré par le mouvement à l'interface) est forcé de faire "demi-tour ", comme montré sur la figure de l'encadré (p. 16) : la pointe est donc le siège d'écoulements qui engendrent une pression, dite de lubrification (cette pression, dans l'huile qui sépare deux pièces solides en mouvement, permet d'éviter le contact : quand les pièces se rapprochent, cette pression augmente). Quand le choc est lent, la pointe est évasée et la pression de lubrification y est négligeable ; mais elle peut devenir critique pour des impacts plus violents. En effet, une augmentation de la vitesse d'impact a pour effet (modeste) d'augmenter la vitesse de l'air et comme conséquence (dramatique) d'accroître le confinement dans la pointe (l'exponentielle de Moffatt). On devine qu'il existe ainsi une vitesse critique (qui dépendra de la viscosité de l'air) au-delà de laquelle la pointe craque sous l'effet de cette pression de lubrification.

La question qui se pose alors concerne la quantité d'air injecté dans le bain. La figure $4 \mathrm{~b}$ et l'image de couverture montrent ce qui se passe juste au-dessus du seuil d'entraînement pour un jet d'huile frappant un bain d'huile : le liquide impactant tire avec lui de l'air qui d'abord le gaine, avant de se fragmenter quelques centimètres plus bas en petites bulles, qui remontent lentement. C'est là ce qui se produit quand un coup de fouet énergique (ou l'action d'un batteur) projette du blanc d'œuf sur lui-même, faisant croître ces blancs en neige. Plus généralement, les mousses seront souvent fabriquées sur ce principe, et connaitre ce qui fixe la quantité d'air permet de comprendre à quelle vitesse la mousse se construit.

La réponse à cette question pourrait (devrait) être très complexe, et bien des problèmes d'entraînement d'une phase fluide par une autre ne sont pas résolus aujourd'hui. Mais dans notre cas, une sorte de simplification remarquable se produit [5]. Tout se comprend par une image, ou plutôt par une comparaison d'images : celles de la figure 4, qui montrent de profil le jet d'huile qui frappe le bain en dessous (fig. 4a) et au-dessus (fig. 4b) du seuil d'entraînement d'air. La différence la plus immédiate est bien sûr, dans le second cas, la présence du film d'air, révélé par le cerne noir qu'il inscrit autour du jet d'huile. La forme du jet retient aussi notre attention : il s'évase en trompette, à cause du ralentissement qu'il subit en s'enfonçant dans le bain. On remarque enfin la façon dont la nappe d'air se résout en petites bulles, quelques centimètres sous la surface du bain (voir aussi l'image de couverture).

Or le point crucial pour le problème qui nous intéresse est ailleurs : c'est la remontée du ménisque à l'endroit de l'impact. Alors que le jet, sous le seuil d'entraînement, s'évase et creuse un trou d'un centimètre environ (et dont la profondeur augmente avec la vitesse), le bain ne s'incurve plus que de quelques millimètres dès que l'entraînement a lieu. En outre, les expériences montrent que cette déformation ne dépend alors plus de la vitesse du jet [6].

Ces faits peuvent s'interpréter en se souvenant du phénomène classique de caléfaction, qui se produit lorsque l'on pose un peu d'eau sur une plaque à $300^{\circ} \mathrm{C}$. Alors le liquide lévite sur la surface, porté par un coussin
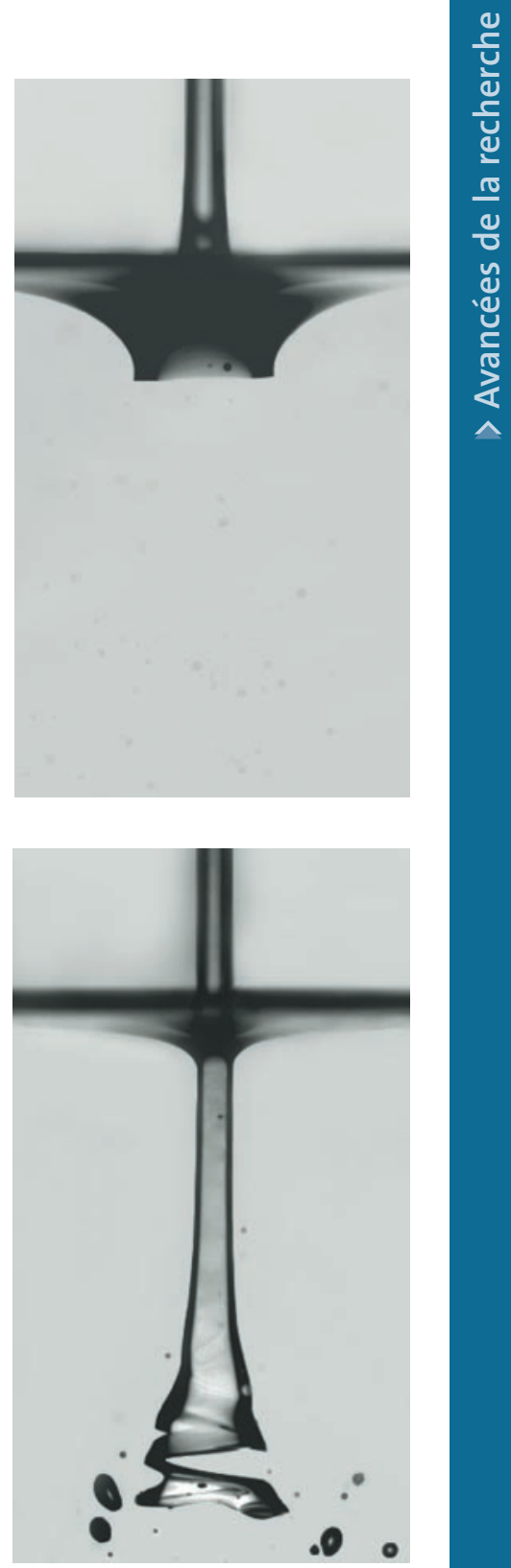

Figure 4 : Jet d'huile silicone (1000 fois plus visqueuse que l'eau) frappant un bain de la même huile. L'échelle des deux photographies est la même, et le diamètre du jet est de 4 millimètres. En haut, la vitesse d'impact n'est pas suffisante pour qu'il $y$ ait entraînement d'air, mais elle creuse néanmoins un trou centimétrique à la surface. On remarque que cette géométrie ne permet pas l'observation de la pointe à la base du creux. En bas, la vitesse d'impact est un peu plus grande, et le jet pénètre dans le bain gainé d'un film d'air. Il s'évase à cause de son ralentissement et quelques centimètres plus bas, la nappe d'air se fragmente en bulles. On remarque que le ménisque fait par le bain contre le jet remonte, par rapport au cas où il n'y a pas entraînement. 


\section{Bibliographie}

[1] D.D. Joseph, J. Nelson,

M. Renardy \& Y. Renardy,

J. Fluid Mech. 223, 383 (1991).

[2] É. Lorenceau, F. Restagno \& D. Quéré, Phys. Rev. Lett. 90,

184501 (2003)

[3] J.T. Jeong \& H.K. Moffatt, J. Fluid Mech. 241, 1 (1992).

[4] J. Eggers, Phys. Rev. Lett. 86, 4290 (2001).

[5] É. Lorenceau, D. Quéré \& J. Eggers, Phys. Rev. Lett. 93, 254501 (2004).

[6] É. Reyssat, Thèse, Université Paris VII (2007).

[7] L. Landau \& V. Levich, Acta physicochimica USSR 17, 42 (1942).

[8] I. Cohen \& S. Nagel, Phys. Rev. Lett. 88, 074501 (2002).

[9] L. Limat, preprint (2007). de vapeur qui lui permet de glisser sur son support. On remarque aussi que le liquide est en mouillage nul, puisque ce n'est plus sur un solide qu'il est posé mais sur de l'air. On peut considérer que l'entraînement d'air (fig. 4b) induit une sorte de caléfaction dynamique : le film d'air, très peu visqueux, " isole " dynamiquement le bain du jet ; en s'interposant, il réduit considérablement l'effet d'entrainement du bain par le jet, rendant le bain quasi-statique. En témoigne la forme de ce dernier à l'endroit de l'impact : on observe un ménisque millimétrique, qui a bien la forme et la taille d'un ménisque statique, et qui est strictement non-mouillant; le bain rejoint là de l'air (l'air du film entraîné), matériau hydrophobe ou oléophobe par excellence, comme nous l'enseigne la caléfaction.

Dès lors, on peut voir la figure $4 \mathrm{~b}$ comme l'analogue (mais tête en bas) de ce qui se passe lorsque l'on retire lentement un solide hors d'un bain mouillant : le solide tire avec lui un peu de liquide, comme ici le jet tire un peu d'air. L'épaisseur de ce film a été calculée par Landau et Levich en 1942 [7]. Elle résulte d'un compromis entre forces visqueuses (qui font l'entraînement) et forces de surfaces (qui s'y opposent, puisque l'entraînement déforme la surface), si bien qu'à nouveau, c'est le nombre capillaire, mais construit cette fois avec la viscosité $\eta_{\mathrm{O}}$ du fluide entraîné (de l'air dans notre cas) qui fixe cette épaisseur. La formule de Landau prévoit que cette épaisseur s'écrit $\kappa^{-1} C a^{2 / 3}$, avec $C a=\eta_{\mathrm{O}} V / \gamma$ et en notant $\kappa^{-1}$ la longueur capillaire (taille du ménisque millimétrique). Pour ces gros jets, on trouve ainsi que le film entraîné fait en général quelques microns d'épaisseur, en excellent accord avec les mesures [5-6].

\section{Autres interfaces singulières}

Les questions de singularités aux interfaces et d'entraînement se posent d'une façon plus générale, bien au-delà de l'exemple traité ici. Parmi les sujets qui intéressent les hydrodynamiciens depuis plusieurs années, nous pouvons ainsi citer l'entraînement sélectif et le dévalement de goutte. Dans le premier cas, exploré par Cohen et Nagel, à Chicago depuis 5 ans, on dispose une pipette au-dessus d'une interface entre deux fluides et on aspire [8]. En augmentant le débit d'aspiration, on voit la déformation de l'interface se durcir, jusqu'à former une pointe, dont le rayon de courbure peut également atteindre le micron. Mais audelà d'un débit critique, la pointe cède et un filament de la phase sous-jacente est émis et entraîné dans la pipette. Pour ce qui est des gouttes dévalant des solides, décrites en particulier par l'équipe de Laurent Limat, on constate là aussi la présence de pointes à l'arrière de ces gouttes qui, quand on augmente leurs vitesses, deviennent de plus en plus marquées, jusqu'à céder en filet quand cette vitesse dépasse une valeur critique [9]. Même si la phénoménologie est semblable dans tous ces exemples, les questions d'entraînement restent en grande part incomprises dans ces deux derniers problèmes, et font aujourd'hui l'objet de recherches actives. Comme dans d'autres sujets non-résolus en hydrodynamique, une des principales difficultés est la coexistence d'échelles de taille très différentes; et d'un point de vue pratique, un des principaux intérêts liés à ces questions est la production, via ces " singularités ", d'objets très petits, souvent difficiles à produire par des moyens plus conventionnels. 